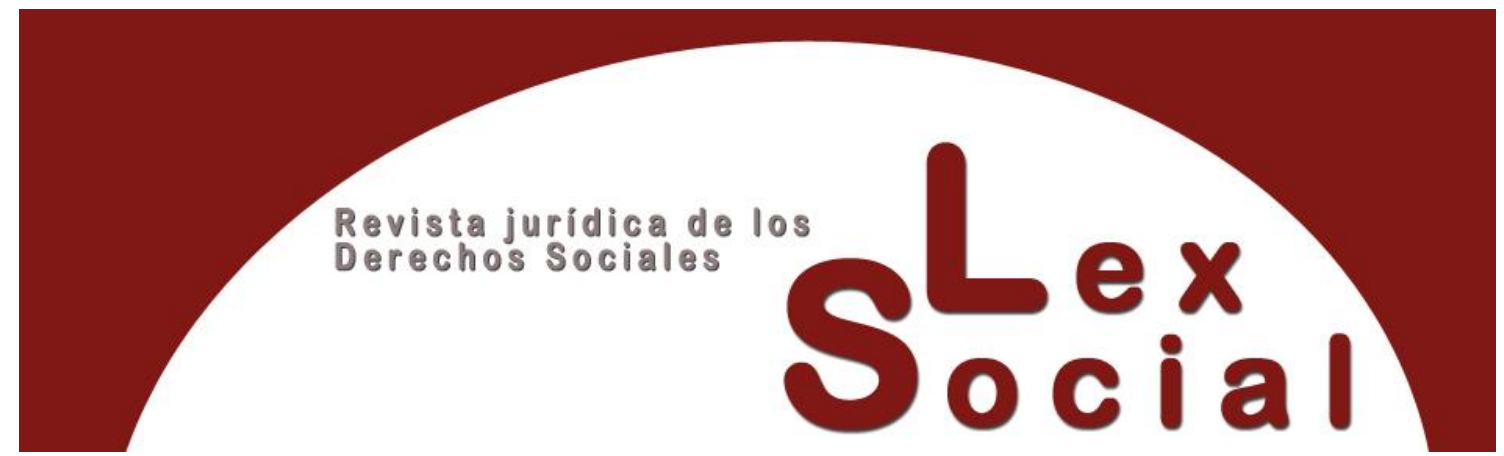

\title{
OBSTÁCULOS DE LA LEGISLACIÓN LABORAL Y DEL RÉGIMEN JURÍDICO DE EXTRANJERÍA EN LA CONSECUCIÓN DE UN TRABAJO DECENTE PARA LAS TRABAJADORAS MIGRANTES DEL SERVICIO DOMÉSTICO
}

\section{OBSTACLES OF LABOUR LAW AND IMMIGRATION LAW TO ACHIEVE A DECENT WORK FOR WOMEN MIGRANT DOMESTIC WORKERS}

FERRAN CAMAS RODA

Catedrático de Derecho del Trabajo y de la Seguridad Social ${ }^{1}$

Universidad de Girona

https://orcid.org/0000-0002-8941-0369000

Cómo citar este trabajo: Camas Roda, F. (2021). Obstáculos de la legislación laboral y del régimen jurídico de extranjería en la consecución de un trabajo decente para las trabajadoras migrantes del servicio doméstico. Lex Social: Revista De Derechos Sociales, 11(1), 449-473. https://doi.org/10.46661/lexsocial.5495

\section{RESUMEN}

Este trabajo tiene por objeto estudiar la normativa laboral sobre el servicio doméstico y del régimen jurídico de extranjería en relación al acceso a España de migrantes para residir y trabajar, en relación a las trabajadoras migrantes que prestan sus servicios para un hogar familiar. La pandemia de la COVID-19 ha hecho del personal de este sector de actividad uno de los más afectados, en particular por las perjudiciales consecuencias que ha tenido para las trabajadoras migrantes, tanto las que tienen declarada su prestación ante las autoridades públicas, como las que trabajan en el sector informal, y dentro de estas, las que están en situación irregular. En este estudio se lanzan propuestas para hacer efectivo respecto a las trabajadoras migrantes del servicio doméstico el principio del Trabajo decente, incorporado como objetivo de

\footnotetext{
${ }^{1}$ Director de la Cátedra de Inmigración, Derechos y Ciudadanía. 
Desarrollo Sostenible en la Agenda 2030 de Naciones Unidas, así como se estudian medidas que mejorarían sus condiciones de trabajo en este ámbito.

Palabras Clave: servicio doméstico; trabajadoras extranjeras; trabajar para el hogar familiar; trabajo decente; Objetivos de Desarrollo Sostenible; sector informal.

\begin{abstract}
The aim of this work is to study the labour Law on domestic workers and immigration Law regarding the rights of migrants to reside and work in Spain, specially Women Migrant Workers, who provide their services for a family home. The COVID-19 pandemic has made the personnel of this sector of activity one of the most affected, in particular due to the damaging consequences it has had for migrant workers, both those who have declared their benefit before the public authorities, as well as the migrants who work in the informal sector, and within these, those who are in an irregular situation. In this study, proposals are launched to make effective with respect to migrant domestic service workers the principle of Decent Work, included as a Sustainable Development Goal in the United Nations 2030 Agenda, as well as measures that would improve their working conditions in this field.
\end{abstract}

KEYWORDS: domestic work; Women Migrant Workers; household work; Decent work; Sustainable Development Goals; informal sector.

SUMARIO

I. Presentación general del estudio: focalización en el colectivo de trabajadoras migrantes e impacto de la COVID-19.

II. Datos de interés sobre el personal del servicio doméstico y las trabajadoras extranjeras afiliadas al sistema de Seguridad Social correspondiente.

III. El Trabajo decente de las trabajadoras migrantes como Objetivo de desarrollo sostenible.

IV. La normativa internacional que vehicula la promoción del Trabajo decente en el empleo de migrantes en el servicio doméstico.

$V$. Regímenes jurídicos en España sobre el personal al servicio del hogar familiar y sobre extranjería: puntos débiles respecto de las trabajadoras migrantes.

1. Cuestiones sensibles de la regulación laboral de carácter especial del hogar familiar.

2. La legislación de extranjería respecto del personal migrante al servicio del hogar familiar. Propuestas de reforma. 
3. Regulaciones relativas a personal migrante del servicio doméstico adoptadas en el marco de la pandemia de la COVID-19.

VI. A modo de conclusiones finales.

Bibliografía.

Anexo.

\section{Presentación general del estudio: focalización en el colectivo de trabajadoras migrantes e impacto de la COVID-19.}

El trabajo en el servicio doméstico, en el que se incluyen tanto labores de cuidado como la prestación de tareas de mantenimiento de un hogar, ha experimentado una transformación muy visible en los años que llevamos de este siglo, en particular la presencia cada vez mayor de trabajadoras migrantes en el marco de un proceso de progresiva sustitución de las mujeres nativas, como al efecto se demostrará posteriormente con los datos sobre ello registrados hasta la fecha. Ello, por cierto, ha tenido efectos positivos en estas últimas, ya que el hecho de que muchas mujeres españolas pudiesen ir delegando, laboralmente hablando, las tareas propias del servicio doméstico a otro personal, sobre todo extranjero, les ha permitido acceder al mercado de trabajo y así aumentar su tasa de actividad en el mercado de trabajo².

En todo caso, las razones de esa incorporación de trabajadoras migrantes al servicio del hogar familiar han sido varias y de raíces diversas. Para empezar, la poca valoración de este tipo de trabajo, que cumple además con las condiciones que tienen los trabajos que, abandonados por los trabajadores nacionales, pasan ser cubiertos por extranjeros, aquellos que cumplen con la regla de las 5-P: pesados, peligrosos, precarios, poco remunerados, y penalizados socialmente ${ }^{3}$. Se ha de decir que el tipo de actividades en los que se han ido concentrando los migrantes ha evolucionado, ya que si en los primeros años del nuevo milenio eran aquellas calificadas como de 3-P, las más penosas, peligrosas y precarias ${ }^{4}$, ahora ha aumentado aún más las franjas ínfimas en condiciones de trabajo que ocupan los trabajadores extranjeros, al añadir a aquellos calificativos las de también ser los peor pagadas, y, partiendo de la base que la antigua categoría de ocupaciones penosas podría incluir a la nueva de pesadas, se añade actualmente también las que son más socialmente minusvaloradas.

Esas características se amoldan al trabajo prestado en el ámbito del servicio doméstico. Por un lado, es escasamente cualificado, socialmente poco valorado, pobremente

\footnotetext{
2 Véase el monográfico incluido en el Informe anual de 2019 del DEFENSOR DEL PUEBLO: La contribución de la inmigración a la economía española: https://www.defensordelpueblo.es/wpcontent/uploads/2020/05/II_Estudios_documentos_de_trabajo_2019.pdf

${ }^{3}$ Véase "Una propuesta sobre un Derecho del Trabajo renovado", de MIGUEL RODRÍGUEZ-PIÑERO Y BRAVO-FERRER, FERNANDO VALDÉS DAL-RÉ, MARÍA EMILIA CASAS BAAMONDE, en la Revista de Derecho de las Relaciones Laborales EDC 2020/660795, comentando lo que a estos afectos también dice el Manifiesto por un Derecho del Trabajo sostenible, de los Profesores italianos Caruso, Del Punta y Treu.

${ }^{4}$ Véase el estudio del año 2004 de L. CACHÓN: “Integración de los inmigrantes en el trabajo", en la revista Documentación Laboral de aquél año.
} 
remunerado y con malas condiciones de trabajo ${ }^{5}$, sobre todo derivadas del poder otorgado al titular del hogar familiar en estas relaciones laborales que puede derivar en arbitrariedades, también por la poca estabilidad y discontinuidad en la prestación de estos trabajos o la simultaneidad en la prestación de servicios para varios hogares que se da por parte de muchas trabajadoras, y naturalmente también por la afectación a la seguridad y salud de las trabajadoras que puede provocar este tipo de trabajo.

Por otro lado, y como al efecto se ha puesto de manifiesto ya por estudios que han tratado esta temática, por resultar un sector cuya base, la de centrarse en un ámbito privado como es el hogar familiar, resulta relativamente propicio para el empleo informal ${ }^{6}$, tanto de trabajadoras españolas como migrantes; es más, esa informalidad se ha erigido como un lugar propicio para que trabajadoras extranjeras en situación irregular hayan visto en él como un puerta de entrada prioritaria al mercado de trabajo, y útil además para solicitar años después una autorización de residencia temporal y trabajo por cuenta ajena a través de las vías excepcionales de arraigo, ya sea el de carácter laboral o el social previstas en la legislación de extranjería.

Ese cúmulo de circunstancias ha resultado fatal para las personas trabajadoras al servicio del hogar familiar ante el impacto de la pandemia de la COVID-19 declarada en el 2020 en el ámbito internacional y también en nuestro país. Como resultado del miedo, la propia situación que podía afectar a los titulares del hogar familiar en sus propios trabajos o condiciones laborales, así como la imposición de confinamientos y la consiguiente restricción de movilidad que impedía que las trabajadoras domésticas llegaran al trabajo, han llevado a que muchas d ellas hayan visto reducidos sus salarios o directamente se hayan visto despojados de ellos, o también hayan visto reducidas sus horas de trabajo y, en muchos casos, la pérdida de sus empleos, al amparo en nuestro país de la posibilidad legal que se concede al titular del hogar familiar para desistir de la relación laboral entablada con la persona trabajadora del servicio del hogar familiar.

De hecho, las estadísticas elaboradas por la Organización Internacional del Trabajo ${ }^{7}$ (en adelante OIT), han puesto de manifiesto como de las consecuencias perjudiciales de carácter laboral de la pandemia no se han librado ni las trabajadoras formales, es decir, las registradas ante autoridades públicas como la Seguridad Social en nuestro país, ni las informales (o dicho de forma general, que disponen de un trabajo no declarado): así, en el ámbito internacional, se detectó en junio de 2020 que un $72 \%$ del personal doméstico había resultado significativamente afectado en su relación de trabajo, de los cuales el 76\% trabajaba de manera informal. Ahora bien, en el norte, sur y este de Europa, la pandemia impactó en el trabajo de solo el $41 \%$ del personal doméstico, si bien el $69 \%$ de éste trabajaba al amparo de un empleo formal.

\footnotetext{
5 Véase el monográfico incluido en el Informe anual de 2019 del DEFENSOR DEL PUEBLO: La contribución de la inmigración a la economía española, ya citada, pp. 40 y ss.

${ }^{6}$ Idem.

${ }^{7}$ INTERNATIONAL LABOUR ORGANIZATION: Impact of the COVID-19 crisis on loss of jobs and hours among domestic workers. 15 / June /2020.
} 
Esas cifras constatan la importancia de la informalidad laboral del servicio doméstico en el ámbito internacional, mucho menor en determinadas zonas de Europa, aunque también testifica que ni siquiera las trabajadoras formales, registradas como tales ante instituciones públicas, han sido impermeables ante la crisis sanitaria y social derivada de la pandemia del coronavirus. No obstante, también se ha de constatar que a diferencia de las informales, las trabajadores con trabajo declarado ante la Seguridad Social han visto reducido los efectos de la crisis sanitaria y económica por diversas medidas gubernamentales. En España, se ha de traer a colación en este sentido la adopción del subsidio extraordinario reconocido a las personas integradas en el Sistema Especial de Empleados de Hogar del Régimen General de la Seguridad Social, por la falta de actividad derivada de la reducción de horas de trabajo, la interrupción de sus servicios por causas ajenas a su voluntad, o la extinción de sus contratos de trabajo por el titular del hogar familiar (véanse los arts. 30 y ss. del Real Decreto-ley 11/2020, de 31 de marzo, por el que se adoptan medidas urgentes complementarias en el ámbito social y económico para hacer frente al COVID-19, posteriormente desarrollado a los efectos de su aplicación por la Resolución de 30 de abril de 2020, del Servicio Público de Empleo Estatal). En todo caso, el reconocimiento excepcional de este subsidio, que habría atenuado la situación personal y social de las personas incluidas en aquellas determinaciones, no debe hacer olvidar que el hecho de que no tengan reconocidas prestaciones de desempleo supone una seria limitación a una protección social adecuada del colectivo del servicio doméstico, también en supuestos de crisis como el que estamos viviendo.

En todo caso, además de esa delimitación entre empleo formal e informal a los efectos de sufrir en mayor o menor medida los efectos sociales y laborales de la COVID-19, lo que no debe perderse de vista es otra diferenciación subyacente a aquella, que además es decisiva respecto a la propia relación de trabajo del personal al servicio doméstico: el derivado de la nacionalidad o el origen étnico de las trabajadoras, es decir, el tratarse de extranjeras o autóctonas. Como se ha advertido por la $\mathrm{OIT}^{8}$, las primeras habrían sufrido situaciones de discriminación en el trabajo, principalmente a través de la finalización de su contrato por los titulares del hogar familiar al haber contraído el virus, lo que las habrías abocado a situaciones de desamparo personal y vital, sin posibilidad además de retornar a sus países por las restricciones a la movilidad internacional, las trabajadoras formales.

Esta situación de discriminación de las trabajadoras migrantes en el servicio doméstico es un factor ciertamente obstativo a la consecución de un trabajo decente para aquellas. Se ha de traerá colación que la Organización Internacional del Trabajo adoptó el Trabajo decente como meta y referente de todas las políticas sociales y económicas de sus miembros, anudándole unos objetivos estratégicos para conseguirlo, en su Declaración sobre la justicia social para una globalización equitativa de 2008. Esos objetivos que constituyen el fundamento del programa sobre Trabajo decente del que se dota la propia OIT para impulsar su plasmación son, por este orden, crear empleo; adoptar y ampliar

\footnotetext{
${ }^{8}$ Idem.
} 
medidas de protección social, incluyendo entre ellas no solo de Seguridad Social, sino también el reconocimiento de condiciones de trabajo seguras y saludables o una cobertura salarial mínima; impulsar el diálogo social como método de aplicación del resto de objetivos; y finalmente, respetar, promover y aplicar los principios y derechos fundamentales en el trabajo9.

En definitiva, el concepto de Trabajo decente que impulsa la OIT constituye un paradigma holístico, es decir, es aplicable a cualesquiera formas de trabajo, entre las que se incluye el servicio doméstico, ya se haga en régimen formal o informal, y naturalmente, ya se haga por personal nativo o extranjero, e incluso en este último caso, tanto en situación regular como irregular por falta de las autorizaciones de residencia o trabajo correspondientes. Como al efecto ha subrayado la OIT en esta cuestión, un principio subyacente al Trabajo decente es el reconocimiento de que todos los que trabajan tienen derechos en el trabajo, cuestión fundamental para reconocer plenamente esa decencia, o mejor, conforme al planteamiento que he realizado en otras de mis publicaciones, la dignidad del trabajo, de las persona que lo realizan y de la comunidad en la que ésta se desarrolla como tal ${ }^{10}$.

Las siguientes páginas van a focalizarse en las trabajadoras migrantes en el servicio doméstico y las medidas legales que obstaculizan su acceso a un trabajo decente. Para ello se partirá se partirá de los datos estadísticos que muestren el nivel de presencia de las trabajadoras extranjeras que prestan servicios para el hogar familiar en nuestro país; posteriormente, se abordarán las iniciativas internacionales a las que están ligadas las políticas públicas en España para potenciar el reconocimiento del trabajo decente: y finalmente, como núcleo fundamental de este trabajo, el análisis de los déficits legislativos, tanto de la legislación de extranjería como de la propia de carácter laboral del servicio doméstico, que dificultarían el reconocimiento de un trabajo decente para las trabajadoras extranjeras. La perspectiva asumida por este estudio parte, en todo caso, de la idea de que su aportación laboral en el ámbito del servicio doméstico será absolutamente necesaria en nuestra sociedad ante la fase histórica de progresivo envejecimiento demográfico en la que se encuentra.

\section{Datos de interés sobre el personal del servicio doméstico y las trabajadoras extranjeras afiliadas al sistema de Seguridad Social correspondiente.}

Antes de abordar las claves del régimen jurídico aplicable al personal al servicio doméstico, conviene conocer que fisonomía tienen dichos sujetos en un año en concreto, el 2019, último a fecha de realización de este escrito que se tiene por completo.

\footnotetext{
${ }^{9}$ Para más información, consúltese Camas Roda, Ferran: Trabajo decente e inmigrantes en España. Un estudio sobre los derechos laborales de los trabajadores migrantes y del objetivo internacional del trabajo decente. Huygens Editorial, Barcelona, 2016.

${ }^{10}$ Idem, pp. 37 y ss.
} 
Para ello, me he dirigido a las estadísticas del Ministerio de Trabajo y Economía Social (MITES), que recoge el número de afiliados al Sistema Especial de Empleados del Hogar, incluido en el Régimen General de Trabajadores. Como después se analizará, se debe avanzar que están incluidos en este sistema aquellos trabajadores que están sujetos a la relación laboral especial del servicio del hogar familiar regulada por el Real Decreto 1620/2011, de 14 de noviembre, excluyéndose en todo caso al personal que preste servicios domésticos no contratados directamente por los titulares del hogar familiar, sino a través de empresas (véase la Disposición adicional decimoséptima de la Ley 27/2011, de 1 de agosto, sobre actualización, adecuación y modernización del sistema de Seguridad Social).

Con esa premisa, conforme a los datos del MITES, en el año 2019 se alcanzó el número de 402,500 personas afiliadas a al Sistema Especial de Empleados del Hogar, de los cuales, 18.000 como varones, y 384.500 como mujeres ${ }^{11}$. Estas cifran permiten visualizar como dicho sistema de protección social está intensamente feminizado, ello tanto si hablamos de nacionales españolas como de extranjeras. En este sentido, si revisamos las estadísticas correspondientes a los trabajadores extranjeros afiliados a la Seguridad Social en alta laboral ${ }^{12}$, resulta que de un conjunto de 170.444 afiliados al Sistema Especial del Hogar Familiar, 10.251 son hombres y 160.179 son mujeres.

Llegados a este punto, conviene hacer tres precisiones de interés sobre la presencia de personal extranjero en este Sistema: en primer lugar, que partiendo de la base que en España se encontraban afiliados a la Seguridad Social en 2019 un total de 2.104.260 extranjeros (1.180.936 varones, y 923.309 mujeres), resulta que el 8\% de no nacionales cotizan en el Sistema Especial del Hogar familiar, si bien el 17\% de las mujeres extranjeras están incluidas en aquel. Es decir, si bien el Sistema del Hogar familiar está feminizado, este se está convirtiendo más bien en un nicho laboral para las trabajadoras extranjeras, y eso solo hablando de aquellas cuyas relaciones están debidamente formalizadas ante la Seguridad Social.

En segundo lugar, y también dirigiendo la vista en las trabajadoras domésticas extranjeras que, como hemos visto, suman un total de 160.179 inscritas en el Sistema correspondiente de la Seguridad Social, el $84 \%$ de ellas tienen entre 30 y 59 años. Si atentemos a la totalidad de afiliadas al Sistema, también resulta que el $81 \%$ de las mujeres está en esa franja. Ahora bien, diferencias de fondo en función de la edad estarían palpitando entre el colectivo de trabajadoras españolas y extranjeras: entre otras, que las trabajadoras extranjeras suelen ser más jóvenes que las españolas, ya que las primeras alcanzan ya el $73 \%$ de afiliadas si nos quedamos con la franja de 30 a 54, mientras que las segundas ya bajan al 65\%. De hecho, la mayor juventud de la población afiliada al Sistema Especial

\footnotetext{
${ }^{11}$ MINISTERIO DE INCLUSIÓN, SEGURIDAD SOCIAL Y MIGRACIONES: AFILIACIONES A LA SEGURIDAD SOCIAL http://www.mites.gob.es/ficheros/ministerio/estadisticas/anuarios/2019/AFI/AFI.pdf ${ }^{12}$ MINISTERIO DE INCLUSIÓN, SEGURIDAD SOCIAL Y MIGRACIONES: AFILIACIONES A LA SEGURIDAD SOCIAL http://www.mites.gob.es/ficheros/ministerio/estadisticas/anuarios/2019/AEX/AEX.pdf
} 
del Hogar Familiar se correspondería con la media de edad que las estadísticas arrojan del conjunto de mujeres extranjeras en el Régimen General, que está en 34,9. Este puede ser otro indicio para sostener que la puerta de acceso al mercado de trabajo para mujeres extranjeras ha venido siendo el servicio del hogar familiar, al igual que también plausible pensar que una parte importante de ellas puede tener nacionalidad de un país del centro o sur de América ya que los mayores porcentajes de mujeres trabajadoras entre el conjunto de afiliados a la Seguridad Social se da en nacionalidades como la paraguaya, la venezolana, la boliviana o la colombiana.

En todo caso, siguiendo con la cuestión de edad, si conforme a los datos estadísticos publicados me centro en la horquilla de 50 hasta 64 años, resulta que mientras que las extranjeras afiliadas son el $31 \%$, el conjunto de mujeres que están incluidas en el sistema son el $44 \%$. En consecuencia, mientras que las trabajadoras extranjeras son más presentes en las primeras franjas de edad (a partir de los 30 sobre todo), y por lo tanto se inician en un Sistema en el que a priori pueden tener recorrido de cotizaciones, el conjunto de mujeres (nacionales o no) que tienen más visibilidad en el sistema lo es a edades más avanzadas (el pico se alcanza entre los 50 a 54 años, con 68.100 afiliadas, mientras que entre los 55 a 59 años llegan a 61.300 personas). Se puede concluir que ahí estarían ingresando mujeres para poder disponer de una carrera de cotización previa a la jubilación.

En tercer y último lugar, no se ha de olvidar que las cifras que he aportado lo son de las personas inscritas en la Seguridad Social, y por tanto, no de las personas nacionales o extranjeras que, aun trabajando en el servicio doméstico, no tienen formalizada su prestación de servicios, es decir, forman parte de la economía informal, y respecto de los extranjeros, tanto si tienen estatuto de residencia como si se encuentra en situación irregular. La informalidad en todo caso se presume alta si se comparan los datos del conjunto de personas afiliadas al Sistema Especial de Seguridad Social, 402,500 como se ha visto anteriormente, con las cifras que publica la Encuesta de Población Activa para 2019 respecto del personal ocupado en hogares empleadores de personal doméstico, que arroja la cifra de 595.200 (de ella, 522.300 son mujeres). Por tanto, conforme a los parámetros diferenciales entre ambas latitudes se constata que podrían existir unas 192.700 personas del servicio doméstico (de ellas, 137.800 mujeres), que pese a prestar sus servicios para hogares empleadores no estarían dadas de alta a la Seguridad Social.

Ese alto nivel de informalidad soportado por este sector de actividad debe ser abordado por los poderes públicos con el objetivo de que, pese a ello, los trabajadores que lo ejercitan dispongan de un mínimo de derechos laborales y de protección social como al efecto pregona el principio de trabajo decente, sobre todo, como vamos a ver a continuación, cuando quienes trabajan en este ámbito del servicio doméstico son trabajadoras migrantes. 


\section{El Trabajo decente de las trabajadoras migrantes como Objetivo de desarrollo sostenible.}

En la Asamblea General de las Naciones Unidas en septiembre 2015 se adoptó la Agenda 2030 de Desarrollo Sostenible ${ }^{13}$, que es un Plan de acción que impulsa dicha organización con el objetivo básico de erradicar la pobreza. A dicho objetivo primero y prioritario, Naciones Unidas le suma otros 16, entre los cuales las cuestiones propias del empleo, del trabajo y de la protección social ocupan un papel destacado. Así, en la propia Introducción de la Resolución se hacen algunas referencias de interés al Trabajo decente ${ }^{14}$, con lo que puede afirmarse que la iniciativa de la OIT pasa a ser asumida por Naciones Unidas de forma que se incorpora como un elemento central de las políticas de sus Estados miembros para implementar la Agenda y de ésta misma para realizar su plan marco de erradicar la pobreza. En este sentido, en la Declaración inicial de Naciones Unidas se expresa su motivación resuelta a crear las condiciones necesarias para crear trabajo decente para todos, teniendo en cuenta los diferentes niveles nacionales de desarrollo y capacidad. También, anticipando lo que van a erigirse como objetivos específicos de la Agenda, en la introducción a la Resolución se erige el compromiso de Naciones Unidas de trabajar para promover el empleo de los jóvenes y el empoderamiento económico de las mujeres, así como el trabajo decente para todos.

Estos compromisos marco encuentran su plasmación posteriormente como Objetivos y metas específicas a nivel mundial, principalmente en Objetivo número 8, que busca "Promover el crecimiento económico sostenido, inclusivo y sostenible, el empleo pleno y productivo y el trabajo decente para todos", reconociéndose así que el acceso a un empleo con un trabajo decente son garantías para salir de la pobreza y construir un modelo de desarrollo sostenible. Dicho objetivo general está compuesto de 12 metas específicas, varias de las cuales quisiera resaltar específicamente a los efectos de este estudio: en primer lugar, la meta 8.5 expresa que con la vista puesta en el 2030 debe lograrse un empleo pleno y productivo y garantizar un trabajo decente "para todas las mujeres y los hombres, incluidos los jóvenes y las personas con discapacidad", y la igualdad de remuneración por trabajo de igual valor. No se cuantifica el número de empleos que deberían crearse, pero si se constatan dos cosas, por un lado, la mención primigenia a las mujeres, por otro el recordatorio que supone citar a los jóvenes y trabajadores con discapacidad como sectores sensibles al desempleo y a la obtención de trabajos decentes.

En segundo lugar, la meta 8.8, que persigue "proteger los derechos laborales y promover un entorno de trabajo seguro y sin riesgos para todos los trabajadores, incluidos los trabajadores migrantes, en particular las mujeres migrantes y las personas con empleos precarios". Debe subrayarse esta meta por cuanto es la única referencia explícita a los migrantes, aunque ello no la hace menos importante; de forma más general, la Agenda

\footnotetext{
13 Resolución aprobada por la Asamblea General el 25 de septiembre de 2015. Naciones Unidas: http://www.un.org/es/comun/docs/?symbol=A/RES/70/1 [Última consulta: 15 de diciembre de 2016].

14 Para un desarrollo de estas cuestiones, consúltese CAMAS RODA, FERRAN: Trabajo decente e inmigrantes en España. Un estudio sobre los derechos laborales de los trabajadores migrantes y del objetivo internacional del trabajo decente, op. cit. pp. 30 y ss.
} 
también aborda cuestiones de interés en materia migratoria, por ejemplo, ya en su misma Introducción al reconocer la positiva contribución de los migrantes al crecimiento inclusivo y al desarrollo sostenible, así como también la necesidad de abordar respuestas coherentes e integrales a la migración internacional; también, ya en el plano de los Objetivos de la Agenda, al prever en el núm. 10, dedicado a "reducir la desigualdad en los países y entre ellos", la meta de recudir hasta el 2030 "a menos del 3\% los costos de transacción de las remesas de los migrantes y eliminar los corredores de remesas con un costo superior al 5\%", lo que denota la importancia también de las economías generadas por los inmigrantes respecto al desarrollo.

A mi modo de ver, la triple conexión entre trabajo decente, migraciones laborales y desarrollo sostenible ampara la realización de políticas y de normativas internacionales y nacionales que promuevan las migraciones laborales de forma ordenada facilitando el acceso de los trabajadores migrantes a los Estados de empleo y respetando sus derechos laborales. Una visión sinóptica de las relaciones de trabajo y de migración en el mundo, que se configure con los valores propios del trabajo decente y de desarrollo sostenible, como son la creación de empleos de calidad, el respeto de derechos laborales y protección social para todos y el interés de la aportación de los migrantes tanto para los Estados de empleo como de origen, impone que se adopten medidas normativas y políticas que favorezcan su movilidad y su integración en el mercado de trabajo al que accedan.

A partir de la especificidad de los trabajadores migrantes en la meta 8.8 configurada por la Agenda 2030 se deriva una implicación especial más; no sólo es que se pida el respeto de los derechos laborales, y en especial la seguridad y salud laboral de los trabajadores migrantes, sino que se hace una mención particular a las mujeres; sin que lo diga, posiblemente la Agenda estaría pensando en la necesidad de reconocer, no únicamente en el principio de igualdad y no discriminación en razón de sexo de las trabajadoras migrantes (aspecto que quedaría incorporado en la meta 8.5 antes referenciada), sino más en concreto en la mayoritaria ocupación que estás disponen en el ámbito internacional en el sector del servicio doméstico. No en vano, en 2013, la OIT dedicó sus Estimaciones mundiales sobre los trabajadores migrantes a tratar de forma específica la cuestión de las trabajadoras y los trabajadores domésticos. En lo que en este último sentido interesa, las Estimaciones constatan en esa anualidad que de 150 millones trabajadores migrantes en el mundo [se ha de decir que en 2018 ya llegaban a 164 millones], 11,5 millones son trabajadores domésticos. Se añadía que, del total de trabajadores migrantes, el 55,7\% son hombres, mientras el 44,3\% son mujeres, si bien la mayor parte de quienes desempeñan trabajos domésticos son estas últimas, alcanzando el 73,4\% (8,5 millones) del total de trabajadores migrantes del servicio doméstico. Finalmente, se constataba que la población trabajadora migrante se concentra en determinados sectores económicos, en concreto el $71,1 \%$ se emplean en el sector servicios, dentro del cual se incluyen los trabajadores migrantes domésticos en un 7,7\%. Si ponemos en conexión el hecho de que 11,5 millones de trabajadores migrantes domésticos junto al dato de que en el mundo se ocupan 67,1 millones de personas en el servicio doméstico, se concluye que, en 2013, de cada seis trabajadores domésticos uno es un migrante internacional. 
Dos conclusiones deben expresarse de esta importante referencia de la Agenda 2030 a los derechos laborales de los trabajadores y trabajadoras migrantes; la primera es el reconocimiento de su situación de vulnerabilidad, en especial en el ámbito laboral ante la falta de protección de sus derechos laborales, por cierto, sin distinción de cuál sea su estatuto, regular o irregular, en el país donde están empleados, aunque como se va a comprobar posteriormente la vulnerabilidad es especialmente intensa en relación a estos últimos.

La segunda es que el objetivo planteado ofrece un argumento importante para superar esa situación de vulnerabilidad que es que las políticas nacionales e internacionales asuman la necesidad de impulsar medidas específicas para ellos. Comentando la meta 8.5, la OIT ha advertido que "los grupos vulnerables, incluyendo los migrantes, requieren una atención especial a fin de garantizar que sean protegidos sus derechos y mejoradas sus condiciones de trabajo"15; en este sentido, esa atención especial debería traducirse en políticas que, sin perder de vista la garantía de un Trabajo decente para todos los trabajadores en las mismas condiciones al margen de su estatuto nacional o de otra índole, fuesen de carácter específico para los migrantes. De forma específica, la meta 8.5 promueve que lo sean en el campo de la prevención de riesgos laborales, cuestión que será tratada posteriormente, pero no debería descartarse la adopción de medidas en el momento del reclutamiento, la contratación o en relación a otras condiciones de trabajo.

\section{La normativa internacional que vehicula la promoción del Trabajo decente en el empleo de migrantes en el servicio doméstico.}

El objetivo de trabajo decente debe ser promovido para todos los trabajadores domésticos en el mundo, incluidos los trabajadores y las trabajadoras migrantes, cuyas necesidades y vulnerabilidades son "específicas", como al efecto reconoce la OIT. Según esta entidad internacional, de forma general el personal que presta sus servicios para un hogar familiar con categoría de empleador dispone de rasgos que lo hacen un colectivo vulnerable, como es el hecho de ser mayoritariamente mujeres o la ejecución de un trabajo en ámbitos privados, lo cual suele hacerlas invisibles, se suma que respecto de las inmigrantes, no suelen estar cubiertas por la legislación laboral vigente en los países donde trabajan o no pueden reclamar esos derechos si trabajan sin documentación ${ }^{16}$.

A la cobertura de estas situaciones tiende el Convenio núm. 189 de la OIT sobre las trabajadoras y los trabajadores domésticos, de 2011, que entró en vigor el 5 de septiembre de 2013, que, por cierto, no ha sido ratificado por España. Este Convenio supone el reconocimiento de cómo algunas de las especificidades propias del personal migrante del servicio doméstico deben ser reguladas a los efectos de su protección. En el articulado del Convenio se recogen de manera exclusiva para el personal extranjero previsiones en

15 OIT: Trabajo Decente y la Agenda 2030 de Desarrollo Sostenible. Estado del Trabajo Decente en el Mundo 2016 (p. 22).

${ }^{16}$ OIT Haciendo el trabajo doméstico visible: el caso de la regulación especial, Ginebra, OIT, 1998. 
relación a derechos de información, como por ejemplo que reciban por escrito, antes de que crucen las fronteras nacionales, una oferta de empleo o un contrato de trabajo que sea ejecutorio en el país donde vayan a prestar sus servicios, o también su derecho a la repatriación tras la expiración o terminación del contrato de trabajo en virtud del cual fueron empleados. En todo caso, los Estados miembros que ratifiquen el Convenio deben adoptar medidas para cooperar entre sí a fin de asegurar la aplicación efectiva de las disposiciones de la norma internacional a los trabajadores domésticos migrantes, así como adoptar medidas específicas para proteger frente a prácticas abusivas a aquellos que hayan sido contratados o colocados por agencias de empleo privadas.

También en relación al personal migrante, la Recomendación sobre las trabajadoras y los trabajadores domésticos, núm. 201, adoptada por la OIT en 2011, se dirige a los Estados miembros para que promuevan la concertación de acuerdos bilaterales, regionales o multilaterales que aseguren que los trabajadores domésticos migrantes amparados por dichos acuerdos gocen de la igualdad de trato con respecto a la seguridad social, así como del acceso a los derechos de seguridad social y al mantenimiento o la transferibilidad de tales derechos.

La normativa derivada tanto del Convenio como de la Recomendación no hace mención específica al estatuto de dichos trabajadores migrantes a los efectos de garantizar su protección; cierto es que el tipo de medidas que el Convenio hace respecto de los trabajadores migrantes haría pensar en la regularidad del personal doméstico admitido a trabajar o en la presión política y jurídica hacia las empresas de colocación para colocar trabajadores bajo las autorizaciones correspondientes. Además, en la Recomendación núm. 201, al dejar la igualdad de trato entre migrantes y personal autóctono a los acuerdos internacionales, también abocaría a pensar en la regularidad de los trabajadores que quisiesen verse amparados por aquellos. En todo caso, debe notarse que muchas de las disposiciones que regulan estas normas hacen referencia a aspectos que tienen que ver directamente con la dignidad de las personas y sus derechos fundamentales en el trabajo, cuyo reconocimiento no podría verse restringido en función de su estatuto, por ejemplo, la referencia que se hace en el art. 9 de la Recomendación para todo trabajador doméstico a que tenga derecho "a conservar sus documentos de viaje y de identidad" u otros que se ajusten a la dignidad personal y los derechos humanos que aparecen listados en dicha norma.

La Recomendación aborda así la protección en forma de servicios de asistencia y ayuda, la previsión de sistema de visitas conforme a los mecanismos de control que tenga establecido el Estado (incluyendo la Inspección de Trabajo), alojamientos, sensibilización empresarial para la proporción de información a los trabajadores sobre las obligaciones legales en materia de empleo e inmigración o el recurso a mecanismos de queja. La Recomendación regula el establecimiento de servicios públicos de asistencia que informe a los trabajadores domésticos, en idiomas que éstos comprendan, sobre sus derechos, así como también expresa la importancia del diálogo social respecto a las condiciones con arreglo a las cuales los trabajadores domésticos migrantes tendrán derecho a ser 
repatriados, sin costo alguno para ellos, tras la expiración o la terminación del contrato de trabajo en virtud del cual fueron empleados, o la promoción de buenas prácticas de las agencias de empleo privadas.

\section{Regímenes jurídicos en España sobre el personal al servicio del hogar familiar y sobre extranjería: puntos débiles respecto de las trabajadoras migrantes.}

El objeto de este apartado es hacer un repaso a dos regímenes jurídicos diferentes, por un lado, el marco jurídico-laboral de la prestación de servicios para el hogar familiar, que tiene por objeto establecer los derechos y deberes de la relación laboral especial que le da el nombre, entre el personal doméstico y el titular del hogar familiar que lo contrata, y por otro, el régimen de extranjería, aplicable a los nacionales de terceros pases a la Unión Europea que pretenden residir y trabajar en España. En todo caso, pese a esa delimitación, este artículo toma como objetivo mencionar aquellos aspectos del primero que en mayor medida pueden afectar a las trabajadoras extranjeras, como también, al tratar el régimen de extranjería, analizaré en qué medida su regulación atiende al sector de actividad del servicio doméstico.

En todo caso, ya avanzo que ambos regímenes, con ópticas diferentes, deberían empezar a escucharse más mutuamente, e interrelacionarse a nivel normativo, para empezar, porque como ya se ha visto, los datos que nos ofrece la realidad de sus trabajadoras abocan a ello.

\section{Cuestiones sensibles de la regulación laboral de carácter especial del hogar familiar.}

La regulación sustantiva de la relación laboral de carácter especial del hogar familiar se encuentra prevista en el Real Decreto 1620/2011, de 14 de noviembre, que considera como tal la que concierta el titular del mismo, como empleador, con el empleado que, dependientemente y por cuenta de aquél, presta servicios retribuidos en el ámbito del hogar familiar. Cabe reseñar que de la aplicación de esa norma se excluyen las relaciones de los cuidadores profesionales contratados por instituciones públicas o por entidades privadas, así como las relaciones de los cuidadores no profesionales consistentes en la atención prestada a personas en situación de dependencia en su domicilio, por personas de la familia o de su entorno, no vinculadas a un servicio de atención profesionalizada, de acuerdo con la Ley 39/2006, 14 de diciembre, de promoción de la autonomía personal y atención a las personas en situación de dependencia.

Esta normativa reglamentaria de carácter laboral pero especial respecto al régimen común de trabajadores por cuenta ajena, establece disposiciones propias para el personal al servicio del hogar familiar en relación a la formalización de contratos, la retribución por sus servicios, el tiempo o jornada de trabajo o en materia de extinción de la relación laboral. En todo caso, en dicha norma no se establece ninguna previsión específica respecto del trabajo que sea desarrollado por personal migrante. Esta cuestión debería replantearse ante la constatación d que una parte importante del trabajo es desarrollada 
por mujeres extranjeras. A ello, se debe sumar el dato de la persistente diferencia en las condiciones de trabajo que vienen arrojando las estadísticas que se publican entre las que disfrutan el personal nativo en el mercado de trabajo y el inmigrante. De forma general, se detecta un mayor índice de temporalidad, de utilización de contratos a tiempo parcial o de subempleo, y en especial de mayor número de horas trabajadas de este último colectivo respeto del nacional ${ }^{17}$. Justamente, en relación con el tiempo de trabajo, los indicadores sociales en España han puesto históricamente de manifiesto la existencia de un mayor número de trabajadores extranjeros que españoles que disponían de un indicador de jornada superior a 40 horas semanales ${ }^{18}$, especialmente constatable en relación a las mujeres, de hecho los sectores con gran concentración de inmigrantes, como hogares que emplean personal doméstico, su indicador de jornada superior a 40 horas ha sido superior en el personal extranjero.

Ante la constatación de que el ámbito del servicio doméstico es cubierto de forma importante por mujeres migrantes, y que éstas suelen percibir más discriminación por cualquiera motivos, empezando por el de nacionalidad, que las propias trabajadoras españolas ${ }^{19}$, considero que deberían arbitrarse medidas para garantizar la eliminación de situaciones de discriminación en casos en que las trabajadoras sean extranjeras. Entre ellas debe destacarse la que empieza a ser conocida como una epidemia propia de este sector de actividad, las situaciones de acoso y abusos de carácter sexual. La necesidad de establecer mecanismos de queja o denuncia específicas para las trabajadoras extranjeras, posterior a la dotación de mecanismos de información sobre ello a través de los servicios públicos o privados de empleo, debería resultar de regulación determinante para evitar esa lacra.

Otra cuestión sensible en la normativa laboral de carácter especial del servicio doméstico que se está analizando es la seguridad y salud de sus trabajadoras. La norma reglamentaria que se está reseñando prevé que el empleador está obligado a cuidar de que el trabajo del empleado de hogar se realice en las debidas condiciones de seguridad y salud, para lo cual adoptará medidas eficaces, teniendo debidamente en cuenta las características específicas del trabajo doméstico. En consecuencia, el reglamento respeta la exclusión de la aplicación de la legislación general de prevención de riesgos laborales, que la propia Ley 31/1995, de 8 de noviembre, en la que se concreta, tiene previsto en su art. 3.4, salvaguardando en todo caso que el titular del hogar familiar está obligado "a cuidar de que el trabajo de sus empleados se realice en las debidas condiciones de seguridad e higiene".

\footnotetext{
${ }^{17}$ Véanse las cifras y ámbitos de diferenciación en las condiciones de trabajo en el informe del Defensor del Pueblo: La contribución de la inmigración a la economía española (publicado en mayo de 2020 e incluido como monográfico específico del Informe anual de Gestión del Defensor del Pueblo en 2019).

${ }^{18}$ Véase por ejemplo el estudio de ARAGÓN MEDINA, Jorge.; MARTÍNEZ POZA, Alicia.; CRUCES

AGUILERA, Jesus.; ROCHA SÁNCHEZ, Fernando.: La integración laboral de las personas inmigrantes en España. Una aproximación al empleo y las condiciones de trabajo. Informes de la Fundación $1^{\circ}$ de Mayo. Junio de 2010. Madrid, 2010, p 113

${ }^{19}$ Véase el estudio de CCOO: L'Ocupació a Catalunya, 2009. CCOO de Catalunya. Juny de 2010. p. 136. 
En general, se ha justificado la inaplicación del régimen legal sobre prevención de riesgos laborales por las dificultades de implementar en la práctica en esta rama de actividad el conjunto de derechos y deberes en materia de protección de la seguridad y salud que se deben implementar en cualquier otro tipo de empresas ${ }^{20}$. En todo caso, la seguridad y salud en el trabajo es concebida en el ámbito internacional como una cuestión que atañe especialmente a los trabajadores migrantes. Los riesgos que derivan de su estatuto migratorio, la ocupación por aquellos de trabajos pesados o penosos como es el servicio doméstico, o el hecho de que quién mayoritariamente lo ocupa son trabajadoras extranjeras, son cuestiones que deberían ser objeto de tratamiento específico en la normativa laboral que les es aplicable. Ello podría suponer que dejase de disponerse de una visión homogénea en materia de seguridad y salud de los trabajadores y trabajadoras al margen de su nacionalidad o lengua, o también calificar su estatuto migratorio como especialmente sensible frente a riesgos laborales en función o no de las dificultades para garantizar adecuadamente la aplicación de medidas de salud en su trabajo.

En materia de extinción de la relación laboral, el Real Decreto 1620/2011 establece que, a diferencia de los trabajadores por cuenta ajena del régimen común, la parte empleadora puede poner fin al contrato por desistimiento, con la única condición a que se comunique por escrito al personal empleado en el hogar, de forma que conste clara e inequívocamente la voluntad del empleador de dar por extinguida la relación laboral por esta causa. Esta posibilidad de desistimiento por voluntad del hogar familiar puede suponer una quiebra en la trayectoria laboral de las trabajadoras extranjeras en situación irregular ya que, al margen de perder el empleo y por tanto su único sustento, les puede quebrar la trayectoria necesaria para obtener una autorización de trabajo por la vía extraordinaria del arraigo social, como al efecto se tratará posteriormente. En todo caso, ya sea tratándose de trabajadoras nativas como extranjeras que trabajen de manera formal, ambas carecen del derecho a obtener una prestación de desempleo que les cubra temporalmente la pérdida de su empleo, lo que como también se tratará en el siguiente apartado, puede implicar serias consecuencias en situaciones de crisis como la que estamos viviendo este año ante la pandemia de la COVID-19. En todo caso, y considerando únicamente la cuestión de la extinción del contrato de trabajo, cabe traer a colación la Sentencia del Tribunal Supremo de 29 de enero de este mismo 2020 (núm. de Recurso: 2401/2017). Se trata del caso de una trabajadora doméstica cesada por desistimiento de la persona empleadora, a la que en instancia se le reconoce el despido como improcedente, y tras recurrirlo en suplicación se le cualifica de nulo con un incremento de indemnización respecto de la sentencia de instancia ya que se acredita que la decisión extintiva se basó en su estado de embarazo. En este sentido, el TS solo entra a resolver, porqué así se lo piden las partes, si la trabajadora tiene derecho a los salarios de tramitación hasta el día de la notificación de la sentencia de suplicación, a lo que responde afirmativamente. No entra a valorar, por tanto,

\footnotetext{
${ }^{20}$ Véase dicha afirmación en la CONFERENCE INTERNATIONALE DU TRAVAIL. 98e session, 2009. Rapport III (Partie 1B). Etude d'ensemble relative à la convention ( $\left.\mathrm{n}^{\mathrm{o}} 155\right)$, à la recommandation $\left(\mathrm{n}^{\circ} 164\right)$ sur la sécurité et la santé des travailleurs, 1981, et au protocole de 2002 relatif à la convention sur la sécurité et la santé au travail, 1981.
} 
sobre si ese tipo de extinción por desistimiento puede ser calificado como nulo, y si a esa calificación le corresponde una indemnización o por el contrario debiera haber supuesto la readmisión de la trabajadora ${ }^{21}$. En todo caso, visto desde el ángulo opuesto, el resultado de todo ello es la validación procesal como despido nulo del desistimiento por la persona empleadora de su trabajadora doméstica, al que se anudan únicamente efectos indemnizatorios con reconocimiento de salarios de tramitación.

En materia de Seguridad Social, el Sistema Especial para Empleados de Hogar encuentra su regulación legal básica en los artículos 250 y 251 del Real Decreto Legislativo 8/2015, de 30 de octubre, por el que se aprueba el texto refundido de la Ley General de la Seguridad Social, que dispensa un tratamiento específico a las empleadas del hogar contratadas por titulares del hogar familiar, a diferencia de las que realicen tareas domésticas al servicio de empresas, que acceden al régimen general de Seguridad Social.

El común denominador de este régimen en materia de actos de encuadramiento, cotizaciones y régimen de prestaciones es que en gran medida los hace descansar en la trabajadora del servicio doméstico, quién además asume principalmente los incumplimientos en aquellos ámbitos, sin que además pueda estar amparada por ningún tipo de asunción de responsabilidades por la Entidad gestora como al efecto sucede en al marco del Régimen general de Seguridad Social. En todo caso, la delegación en las trabajadoras de la ejecución de actos formales correspondientes a su prestación de servicios sucede en prestaciones de servicios de jornada laboral breve, para uno o varios empleadores, que supone una porción trascendente del régimen de empleadas del hogar al que acceden trabajadoras extranjeras. Así, por ejemplo, conforme al Real Decreto-ley 29/2012, de 28 de diciembre, el personal doméstico que preste sus servicios durante menos de 60 horas mensuales por empleador deberá formular directamente su afiliación, altas, bajas y variaciones de datos cuando así lo acuerden con los empleadores que pueda tener, los cuales, en todo caso, deberán firmar aquellos actos de encuadramiento; además, el propio personal del hogar familiar son los sujetos responsables de la obligación de cotizar al sistema, incluyendo las cuotas por las cotizaciones que deba pagar el empleador. En todo caso, pese a que la normativa delegue en el propio personal la realización de estos actos formales, no por ello inadmite la intervención del empleador, facilitando a éstos que con un solo acto administrativo se pueda dar por cumplida varios, v. gr., la comunicación de un titular del hogar familiar de la alta o de la baja en la Seguridad Social de su personal doméstico supone automáticamente el cumplimiento del deber de comunicar al Servicio Público de Empleo el contrato realizado, o bien de su terminación (vid., Disposición adicional primera del Real Decreto 1620/2011).

\footnotetext{
${ }^{21}$ Respecto del debate a cómo proceder en caso de despidos nulos en los supuestos de relaciones laborales especiales del servicio del hogar familiar, véase M. Inmaculada BENAVENTE TORRES: Un aspecto jurídico especialmente problemático: la extinción de la relación laboral, en Revista Jurídica de los Derechos Sociales, Lex Social, 2019. La autora mantiene que en los despidos disciplinarios se pueda declarar la nulidad pero con efectos restringidos a una indemnización, salarios de tramitación e indemnizaciones por vulneración de Derechos fundamentales (p. 271).
} 
El hecho de que la normativa atribuyan a las empleadas del hogar un papel preponderante en la formalización de actos a la Seguridad Social en aquellas prestaciones de servicio inferiores a las 60 horas mensuales supone a mi parecer una pista favorecedora de la informalidad, tanto para las trabajadoras nativas como para las extranjeras. De hecho, a estas últimas se le puede añadir el obstáculo de no dominar el idioma o los mecanismos institucionales para canalizar la formalización de su relación laboral, lo cual puede suponer ser pasto de abusos en los intermediarios para realizarlas. Debería pensarse en la creación de mecanismos públicos de asistencia e información respecto de las trabajadoras domésticas sobre sus derechos de carácter formal ${ }^{22}$.

\section{La legislación de extranjería respecto del personal migrante al servicio del hogar familiar. Propuestas de reforma.}

El marco normativo en materia de extranjería compuesto por la Ley Orgánica 4/2000, de 11 de enero, sobre derechos y libertades de los extranjeros en España y su integración social, y el reglamento que la desarrolla, el Real Decreto 557/2011, de 20 de abril, no disponen de un tratamiento específico ya sea respecto de los derechos y deberes del personal del servicio doméstico, ya sea respecto de los procedimientos en los que pueden estar inmersos, más allá de una referencia al pago de las tasas que deben se deben realizar por la tramitación de las autorizaciones de residencia y de trabajo (véase arts. 45 y 46 de la Ley Orgánica 4/2000 en relación con la Disposición adicional decimoctava del reglamento).

Desde hace años, tampoco en el Catálogo de Ocupaciones de Difícil Cobertura regulado en el art. 65 del Real Decreto 557/2011, que facilita la superación por los extranjeros que no se hallan en nuestro país del condicionante de la situación nacional de empleo y así poder acceder a España para trabajar, se recoge ninguna mención a la necesidad de personal doméstico en el mercado de trabajo. Así, por ejemplo, en la Resolución de 13 de octubre de 2020, del Servicio Público de Empleo Estatal, por la que se publica el Catálogo de Ocupaciones de Difícil Cobertura para el cuarto trimestre de 2020, únicamente se incluyen aquellas relacionadas con el sector de Marina Mercante, así como las relativas a entrenadores y deportistas profesionales.

Quisiera destacar esta cerrazón de la normativa española a la recepción de personal extranjero para trabajar en el servicio doméstico frente a lo que ha ocurrido durante este 2020 tras la declaración del primer estado de alarma del mes de marzo frente a la crisis sanitaria derivada de la pandemia del coronavirus. Entre otras regulaciones que derivaron de dicha alarma, se adoptó el Real Decreto-ley 10/2020, de 29 de marzo, por el que se

\footnotetext{
${ }^{22}$ En un trabajo realizado por los profesores. Eduardo ROJO, Ferran CAMAS E Ignacio CAMÓS, titulado: Informe sobre la regulación laboral y de Seguridad Social de la prestación de servicios en el hogar familiar. Propuestas de reforma, presentado a la entidad EMERGIM, en el año 2004, los autores ya hacían hincapié en la necesidad de promover campañas en este sentido. Concluían que debería producirse una amplia campaña de explicación al conjunto de trabajadores domésticos, y muy en especial a los inmigrantes, sobre los derechos y deberes en materia laboral y de protección social, junto a una revalorización social del trabajo doméstico.
} 
regula un permiso retribuido recuperable para las personas trabajadoras por cuenta ajena que no presten servicios esenciales, con el fin de reducir la movilidad de la población en el contexto de la lucha contra el COVID-19. El objeto de esta legislación era otorgar un permiso retribuido a los trabajadores recuperable, de carácter obligatorio, entre el 30 de marzo y el 9 de abril de 2020, exceptuando aquellos que prestasen servicios en los sectores calificados como esenciales en el anexo que se adjuntaba a dicho real decreto-ley. Es decir, por primera vez se listaban unos sectores calificándolos de esenciales, en función de los cuales las actividades en ellos incluidas debían seguir ejecutándose pese a la epidemia virológica, es decir, las personas trabajadoras debían seguir prestando sus servicios en ellas pese a la declaración del estado de alarma. Entre esas actividades esenciales se encontraban, entre otros, los establecimientos comerciales de alimentación; las lavanderías; las de la restauración que prestan servicios de entregas a domicilio, así como también, así como las personas que atiendan mayores, menores, dependientes o con discapacidad.

Ese calificativo de esencialidad se hizo sobre una tipología de sectores y actividades cubiertas por personal con relaciones laborales en muchos casos precarias, lo cual merecería una reflexión sobre la necesidad de dotar a dichos trabajadores de empleos de calidad. Además, muchas de dichas ocupaciones han estado ocupadas por personal migrante. En este sentido, debe traerse a colación que, como media en el conjunto de los Estados miembros, el $13 \%$ de los trabajadores en «profesiones clave» son migrantes, y que los migrantes extracomunitarios se encuentran sobrerrepresentados en empleos de servicios esenciales de primera línea en sectores como la asistencia sanitaria, la alimentación, la distribución y el transporte ${ }^{23}$.

A mi parecer, además de lo dicho sobre la necesidad de focalizar políticas laborales que implementen trabajos de calidad en dichos sectores esenciales, también debería resolverse el desajuste que se puede producir entre la restricción existente sobre la permisión de acceso al mercado de trabajo español de extranjeros no residentes en España por una parte, y la calificación de esencialidad de determinadas actividades productivas y laborales en España. De hecho, hay que recordar que el Catálogo de ocupaciones de difícil cobertura, que permite otorgar autorizaciones a extranjeros para que vengan a España a trabajar en los empleos que lista (este año, entrenadores deportivos y trabajos en la marina mercante), no lo hace por tanto con empleos que son esenciales en nuestra sociedad, especialmente en el ámbito de los cuidados y atención de personas a domicilio. De hecho, en la lista de dicho catálogo ninguna de las ocupaciones que contiene se corresponde con lo que después ha resultado ser esencial, lo que debería ser objeto de replanteamiento.

La actividad correspondiente al sector de cuidados y atención a personas en dependencia, mayores, menores o con discapacidades, que va mucho más allá del específico régimen laboral del personal del hogar familiar, aunque lo engloba también, está llamado a ser fundamental en nuestra sociedad, no solo ante las actuales circunstancias que lo han hecho

\footnotetext{
${ }^{23}$ Véase el Dictamen de la Sección de Empleo, Asuntos Sociales y Ciudadanía del CESE: La gestión de la diversidad en los Estados miembros de la UE, de 9 de septiembre de 2020.
} 
esencial, sino también ante el proceso de progresivo envejecimiento en el que estamos inmersos. Por esa razón, considero que deben abrirse las vías legales para acceder a España a trabajar en el que se tenga en cuenta este sector del mercado de trabajo, entre otras vías valorando también la reformulación del Este catálogo de oficios permite que extranjeros que quieren ocuparlas vengan a trabajar a España al amparo de una autorización de residencia y trabajo. Tal vez sería hora de abrir la posibilidad de completar y dotar mejor las actividades esenciales con personas extranjeras que quieran venir a ocupar los trabajos en España, o a aquellas que, pese a hacerlo de manera irregular, estén trabajando ya aquí en el ámbito del empleo del hogar familiar, algunas de cuyas funciones entrarían dentro de la calificación de actividades esenciales, puedan obtener la correspondiente autorización.

Además de lo anterior, es necesario también traer a colación determinadas iniciativas para la reforma de la legislación de extranjería donde se encuentran involucradas las trabajadoras extranjeras del servicio doméstico. Esas iniciativa han sido lanzadas por el Defensor del Pueblo en sus informes anuales, en el último de los cuales, previo a la pandemia de la COVID-19, se adjuntó un informe sobre La contribución de la inmigración a la economía española, en la que se encuentran varias propuestas de reforma ${ }^{24}$.

Especialmente interesantes son varias de las recomendaciones que afectan a la autorización de residencia temporal y trabajo por cuenta ajena. Aunque se trata de propuestas cuya adopción afectaría al conjunto de personas trabajadoras que pretendan obtener dicho permiso, el Defensor del Pueblo las desarrolla tras el tratamiento que hace en su estudio sobre las problemáticas del sector de cuidados y del servicio doméstico. Es decir, se trata de recomendaciones de reforma normativa que tienen como marco de referencia a las actividades propias del servicio del hogar familiar, y, por tanto, su aceptación tendría un fuerte impacto en este régimen.

Para empezar, la necesidad de modular las cantidades económicas que están fijadas en el arts. 66.2a) del Real Decreto 557/2011 y que cualquier empleador debe acreditar para justificar que cuenta con medios económicos suficientes a los efectos de formalizar el contrato de trabajo gracias al cual se obtenga la autorización. Al margen de la petición de esa modulación sobre los porcentajes económicos que se establecen en aquel precepto, no añade nada el Defensor del Pueblo, aunque considero que más que de modulación, lo que se debería hacer es establecer de forma expresa una regulación específica sobre dichas cantidades, al efecto de rebajar en lo posible su cuantía cuando el empleador lo es un titular del servicio del hogar familiar, sobre todo en el caso de que se trate de unidades de dos o más personas. En este sector de actividad, también se proveen servicios las empresas de cuidados, aunque considero que la mayor flexibilidad moduladora debería centrarse cuando quién contrata es un titular del hogar familiar, arbitrando las medidas ${ }^{24}$ DEFENSOR DEL PUEBLO: La contribución de la inmigración a la economía española, obra ya citada
anteriormente, pp. 40 y ss. 
necesarias para sus beneficiarios de las medidas necesarias para que respeten la normativa de extranjería y de carácter laboral.

También, de forma general respecto del procedimiento de solicitud de autorización inicial y residencia y trabajo por cuenta ajena, aunque no perdiendo la vista a la importancia que ello puede tener para las trabajadoras extranjeras del hogar familiar, el Defensor del Pueblo pide ampliar el plazo establecido en el artículo 67.8 del Real Decreto 55/2011, en caso de fallecimiento del empleador, para que el trabajador pueda ser dado de alta por otro empleador, que ahora se limita a tres meses desde la entrada del extranjero en España.

En todo caso, donde el Defensor del Pueblo si hace una mención específica al régimen especial de empleadas del hogar es al recomendar una recomendación normativa en materia de autorizaciones de residencia por reagrupación familiar. En este sentido, aboga por modificar el artículo 54 del Real Decreto 557/2011, minorando las exigencias económicas fijadas conforme al IPREM en los casos de extranjeros reagrupantes que estén dados de alta en el régimen especial de empleados del hogar, con hijos menores a su cargo en el país de origen.

\section{Regulaciones relativas a personal migrante del servicio doméstico adoptadas en el marco de la pandemia de la COVID-19.}

La actividad legislativa o administrativa respecto del personal extranjero que presta sus servicios para el hogar familiar se ha manifestado en los siguientes aspectos:

1. Se ha de reseñar la Primera Instrucción sobre la Renovación de las autorizaciones de residencia temporal y trabajo por cuenta ajena de la Dirección General de Migraciones, incluida dentro de las Instrucciones DGM 5/2020, que aquella publicó con fecha de 8 de junio de $2020^{25}$.

Se trata de una instrucción que en materia de renovación de las autorizaciones de residencia temporal y trabajo por cuenta ajena parte de considerar la aplicación del procedimiento previsto en el artículo 71, con los efectos del artículo 72 del Real Decreto 55//2011. En este sentido, uno de los supuestos que habilita la renovación consiste en acreditar "la continuidad en la relación laboral que dio lugar a la concesión de la autorización cuya renovación se pretende", la Instrucción señala que dicha continuidad se mantiene en los casos en los que la persona extranjera se encuentre dada de alta en el Sistema Especial de Empleados de Hogar del Régimen General de la Seguridad Social y se haya producido una reducción, total o parcial, en su jornada laboral.

Por otra parte, otro de los supuestos que también fundamentan la renovación de las autorizaciones residencia temporal y trabajo por cuenta ajena consisten en que, al finalizar el período de la autorización que se presente renovar, la persona extranjera tenga atribuida

25 Véanse las INSTRUCCIONES DGM 5/2020 SOBRE LA RENOVACIÓN DE LAS AUTORIZACIONES DE RESIDENCIA Y/O TRABAJO EN EL CONTEXTO DEL COVID 19. Ministerio de Inclusión, Seguridad Social y http://extranjeros.inclusion.gob.es/ficheros/normativa/nacional/instrucciones_sgie/documentos/2020/INS TRUCCION_renovaciones.pdf 
una prestación contributiva por desempleo, o bien cuando sea beneficiaria de una prestación económica asistencial de carácter público destinada a lograr su inserción social o laboral (art. 7.1.2d) del Real Decreto 557/2011 en relación con los arts. 38.6b) y c) de la LO 4/2000). A lso efectos de renovar las correspondientes autorizaciones, la Instrucción que se está comentando incluye dentro de dichos preceptos, "aquellas otras prestaciones que puedan implantarse, el ingreso mínimo vital, el subsidio de desempleo excepcional por fin de contrato temporal y subsidio extraordinario por falta de actividad para las personas integradas en el Sistema Especial para Empleados de Hogar del Régimen General de la Seguridad Social".

2. La Instrucción cuarta de las Instrucciones DGM 5/2020 que se están referenciando tienen por objeto abordar las renovaciones de las autorizaciones de residencia en virtud de reagrupación familiar. De hecho, en el art. 61 del Real Decreto 557/2011 fija como requisitos que el reagrupante ha de cumplir el que sea titular de una autorización de residencia en vigor, que cuente con empleo y/o recursos económicos suficientes para atender las necesidades de la familia en una cantidad que represente mensualmente el 100\% del IPREM, computándose a dichos efectos los ingresos provenientes del sistema de asistencia social; así como también que disponga de una vivienda adecuada para atender sus necesidades y las de su familia, y que habrá de ser su vivienda habitual.

En el aspecto relativo al empleo, dice la Instrucción cuarta que se entenderá cumplido en aquellos casos en los que el reagrupante (o su cónyuge o pareja en los supuestos de reagrupación establecidos en el art. 61 del Real Decreto 557/2011), se encuentre dado de alta en el Sistema Especial de Empleados de Hogar del Régimen General de la Seguridad Social y se haya producido una reducción, total o parcial, en su jornada laboral.

3. Finalmente, cabe traer a colación las Instrucciones DGM 6/2020 sobre los procedimientos iniciados relativos a arraigos sociales en el contexto del COVID-19, publicadas con fecha de 8 de junio de $2020^{26}$. En lo que a nuestro objeto de estudio importa, estas instrucciones abordan las solicitudes de autorización de residencia temporal por razones de arraigo social, que están reguladas en el artículo 124.2 del Real Decreto 557/2011, en particular respecto de aquellas solicitudes en las que se aporte un informe que exima al extranjero que pretende arraigarse de contar con un contrato de trabajo, para lo cual se debe proceder a valorar la acreditación de que cuenta con medios económicos para residir temporalmente en España.

En este aspecto, la Instrucción señala en relación con la suficiencia de medios económicos, deberá procederse a efectuar una valoración no tasada de las circunstancias concurrentes en cada caso, así, podría valorarse entre otros elementos la existencia de un contrato de trabajo o del desarrollo de una actividad por cuenta propia y la estabilidad de estos como fuentes de recursos en unos términos determinados en la instrucción. En este

26 Véase la página web del MINISTERIO DE INCLUSIÓN, SEGURIDAD Y MIGRACIONES: http://extranjeros.inclusion.gob.es/ficheros/normativa/nacional/instrucciones_sgie/documentos/2020/INS TRUCCION iniciales.pdf 
sentido, se confirmaría que existe un contrato de trabajo (y, por tanto, hay estabilidad), en aquellos en los que el familiar haya sufrido una reducción, total o parcial, de la jornada en relación con personas integradas en el Sistema Especial para Empleados de Hogar del Régimen General de la Seguridad Social.

La Instrucción señala que a los efectos de valorar los recursos económicos del reagrupante, también se tendrán en consideración el reconocimiento y/o percepción del ingreso mínimo vital, así como el subsidio extraordinario por falta de actividad para las personas integradas en el Sistema Especial para Empleados de Hogar del Régimen General de la Seguridad Social.

4. Finalmente, se ha de reseñar la iniciativa desarrollada por la Comunidad Autónoma de Cataluña, concretada en la aprobación del Decreto Ley 25/2020, de 16 de junio, de medidas extraordinarias en materia social y de carácter fiscal y administrativo. En su art. 1 se crea una subvención específicamente dirigida a la "creación de nuevo empleo" en el ámbito de los cuidados. De hecho, la subvención se concreta en una prestación económica dirigida a las personas empleadoras o a las entidades sin ánimo de lucro, con el objetivo de promover el empleo en el ámbito de la atención a las personas mayores o dependientes. Lo determinante de esta nueva subvención es que se concederá a los sujetos beneficiarios que ofrezcan un contrato laboral para hacer trabajos de cuidado de personas mayores o dependientes por un mínimo de 12 meses a personas que no hayan sido dadas de alta en la seguridad Social en los últimos 2 años a contar desde la fecha de firma del contrato o a personas que cumplan los requisitos establecidos para poder obtener una autorización de trabajo por arraigo social conforme a lo previsto en el artículo 124.2 del Real Decreto 557/2011.

En consecuencia, pasa a otorgarse una prestación económica a personas empleadoras para que, o bien hagan emerger trabajo informal en el sector de cuidados, o bien tengan a su servicio personas extranjeras en situación irregular que cumplan con los requisitos para arraigarse socialmente y con ello obtener una autorización de residencia temporal y trabajo por cuenta ajena, entre los cuales estará justamente la acreditación de la relación laboral de un año como mínimo.

Esta iniciativa me parece muy interesante, si bien, pese a tratarse de una política que buscaría paliar en determinada medida los efectos de la crisis derivada de la pandemia de la COVID-19, el inmenso alcance de ésta puede haber sido un impedimento para reducir los efectos de aquella política. La ayuda destinada a los titulares del hogar familiar, no a las propias trabajadoras inmigrantes regulares, pero con trabajo no declarado, o sometidas a situación irregular, puede haber tenido efectos limitados cuando, como se ha visto anteriormente, la crisis sanitaria y económica se ha cebado sobre todo con dichas mujeres respecto a la reducción de horas de trabajo o extinción de sus contratos. Es decir, se subvenciona a empleadores cuando los datos arrojan que han tomado un número importante de decisiones de extinción de las relaciones laborales del servicio del hogar familiar respecto a trabajadoras informales o irregulares, y se deja sin cobertura social las que han sufrido principalmente aquellas decisiones. 


\section{A modo de conclusiones finales.}

Este trabajo ha pretendido mostrar el estado, en términos jurídicos y sociales, de la presencia de las trabajadoras extranjeras al servicio del hogar familiar, principalmente con el desencadenante de la pandemia del coronavirus SARS-CoV-2, y sus derivadas sanitarias y económicas. Con esos datos sobre la mesa, debe confirmarse que tanto el régimen laboral del servicio del hogar familiar, como el propio régimen de extranjería, deben abordar de forma específica el acceso a España para trabajar, por una parte, pero también las condiciones de trabajo y de protección social de las trabajadoras migrantes que prestan servicios en ese sector.

Ese abordaje debería realizarse teniendo en cuenta los parámetros que derivan del principio o guía sobre Trabajo decente que, impulsado por la Organización Internacional del Trabajo primero, ha tenido su reconcomiendo como objetivo de Desarrollo Sostenible en la Agenda 2030 de Naciones Unidas, el cual, por cierto, toma como colectivo al que dirigirse de forma especial, el de las mujeres migrantes, lo cual sitúa al servicio doméstico como uno de los ámbitos donde con mayor relevancia deben aplicarse los programas destinados a cumplir con aquel principio.

Como ha sido objeto de comentario anteriormente, esa atención especial al servicio doméstico por estar ocupado en gran medida por trabajadoras extranjeras, debería empezar en España por ratificar el Convenio núm. 189 de la OIT sobre las trabajadoras y los trabajadores domésticos, de 2011 y consideración de lo dispuesto en la Recomendación sobre las trabajadoras y los trabajadores domésticos, núm. 201 de 2011. Además de ello, en todo caso, la normativa social ha de dotarse de instrumentos especiales en materia de lucha contra la discriminación y abusos o acosos de carácter sexual, seguridad y salud laboral, contratación laboral, promoción de la formalización de las relaciones de trabajo concertadas, condiciones de trabajo y protección social en general, que cubriesen tanto las trabajadoras extranjeras en situación regular como irregular. Para ello, seria de interés potenciar que la regulación actualmente existente sobre la relación laboral especial del hogar familiar incluyese menciones específicas a las trabajadoras extranjeras a los efectos de tener en cuenta sus especiales características como tales.

Desde el punto de vista de la legislación de extranjería, seria de interés también que se asumiese una normativa especial respecto del acceso a España de estas para trabajar. Las propuestas que están sobre la mesa han sido objeto de comentario anteriormente, pero no por ello se ha de insistir en un cambio de óptica respecto a la necesidad de reconfigurar las vías legales actualmente existentes a los efectos de crear una de carácter específico para el sector de los cuidados. El cambio demográfico ya está aquí, haciendo que nuestro país sea uno de los más longevos en esperanza de vida (83.5 años de vida en 2070), y con menor índice de fecundidad (aprox. 1.25 /mujer), por debajo del valor de 2.1 que se considera como el requerido para mantener el tamaño de la población constante en ausencia de migración. Esa situación obliga a replantear el formato de un sector que sigue promoviendo la informalidad de las trabajadoras y la irregularidad administrativas de las extranjeras. 
Para ello, es necesario un diálogo entre ambos regímenes jurídicos, el de extranjería y el laboral en materia de servicio doméstico, es decir, que en el ámbito jurídico laboral del servicio doméstico se tuviese en cuenta la especificidad de las trabajadoras migrantes, mientras que en el ámbito de extranjería se tuviese en cuenta el nicho de empleo que representan los trabajos de cuidados y al servicio del hogar familiar. Esa ignorancia mutua entre ambos regímenes habría resultado letal ante la pandemia de la COVID-19, cuyas ondas expansivas han seguido varias direcciones: por un lado, el desamparo social de muchas trabajadoras extranjeras en situación irregular ante la finalización de su trabajo, con serio quebranto tanto para su supervivencia como para la obtención del anhelado estatuto de residencia; y por otro, la falta de cobertura de aquellas que, aun residiendo legalmente, trabajaban de manera informal, imposibilitándose con ello la recepción de cualquier tipo de ayuda.

El régimen jurídico laboral del empleo del hogar familiar debe tener en consideración las peculiaridades de las trabajadoras extranjeras, mientras que la legislación de extranjería debe arbitrar menciones específicas aplicables a las migrantes empleadas en aquél ámbito. No hacerlo solo conseguirá aumentar las solfataras que siguen emanando del terreno jurídico del servicio doméstico.

\section{Bibliografía.}

ARAGÓN MEDINA, Jorge; MARTÍNEZ POZA, Alicia; CRUCES AGUILERA, Jesus.; ROCHA SÁNCHEZ, Fernando.: La integración laboral de las personas inmigrantes en España. Una aproximación al empleo y las condiciones de trabajo. Informes de la Fundación $1^{\circ}$ de Mayo. Junio de 2010. Madrid, 2010, p 113

BENAVENTE TORRE, M. Inmaculada: "Un aspecto jurídico especialmente problemático: la extinción de la relación laboral”,Revista Jurídica de los Derechos Sociales, Lex Social, 2019.3

CACHÓN, Lorenzo. "Integración de los inmigrantes en el trabajo", en la revista Documentación Laboral, 2003.

CAMAS RODA, Ferran: Trabajo decente e inmigrantes en España. Un estudio sobre los derechos laborales de los trabajadores migrantes y del objetivo internacional del trabajo decente. Huygens Editorial, Barcelona, 2016.

RODRÍGUEZ-PIÑERO Y BRAVO-FERRER, Miguel, VALDÉS DAL-RÉ, Fernando, Y CASAS BAAMONDE, María Emilia: Una propuesta sobre un Derecho del Trabajo renovado", en la Revista de Derecho de las Relaciones Laborales EDC 2020/660795.

ROJO, Eduardo, CAMAS, Ferran, Y CAMÓS, Ignacio. Informe sobre la regulación laboral y de Seguridad Social de la prestación de servicios en el hogar familiar. Propuestas de reforma, presentado a la entidad EMERGIM, en el año 2004 (material inédito).

\section{Anexo.}


CCOO: L'Ocupació a Catalunya, 2009. CCOO de Catalunya. Juny de 2010. p. 136.

CESE: Dictamen de la Sección de Empleo, Asuntos Sociales y Ciudadanía del CESE: La gestión de la diversidad en los Estados miembros de la UE, de 9 de septiembre de 2020.

CONFERENCE INTERNATIONALE DU TRAVAIL. 98e session, 2009. Rapport III (Partie 1B). Etude d'ensemble relative à la convention ( $\left.\mathrm{n}^{\circ} 155\right)$, à la recommandation $\left(\mathrm{n}^{\circ}\right.$ 164) sur la sécurité et la santé des travailleurs, 1981, et au protocole de 2002 relatif à la convention sur la sécurité et la santé au travail, 1981.

DEFENSOR DEL PUEBLO: Informe anual de 2019 del Defensor del Pueblo: La contribución de la inmigración a la economía española : https://www.defensordelpueblo.es/wpcontent/uploads/2020/05/II_Estudios_documentos_de_trabajo_2019.pdf

INTERNATIONAL LABOUR ORGANIZATION: Impact of the COVID-19 crisis on loss of jobs and hours among domestic workers. 15 / June /2020.

INSTRUCCIONES DGM 5/2020 SOBRE LA RENOVACIÓN DE LAS AUTORIZACIONES DE RESIDENCIA Y/O TRABAJO EN EL CONTEXTO DEL COVID 19. Ministerio de Inclusión, Seguridad Social y Migraciones: http://extranjeros.inclusion.gob.es/ficheros/normativa/nacional/instrucciones_sgie/documentos/2020/INSTRUCCION renovaciones.pdf

MINISTERIO DE INCLUSIÓN, SEGURIDAD SOCIAL Y MIGRACIONES: AFILIACIONES A LA SEGURIDAD SOCIAL: http://www.mites.gob.es/ficheros/ministerio/estadisticas/anuarios/2019/AFI/AFI.pdf

MINISTERIO DE INCLUSIÓN, SEGURIDAD SOCIAL Y MIGRACIONES . TRABAJADORES EXTRANJEROS AFILIADOS A LA SEGURIDAD SOCIAL: http://www.mites.gob.es/ficheros/ministerio/estadisticas/anuarios/2019/AEX/AEX.pdf

OIT Haciendo el trabajo doméstico visible: el caso de la regulación especial, Ginebra, OIT, 1998.

OIT: Trabajo Decente y la Agenda 2030 de Desarrollo Sostenible. Estado del Trabajo Decente en el Mundo 2016. 